\title{
THEORETICAL ANALYSIS OF BLOWDOWN PROCESS IN CHINA HELIUM-COOLED SOLID BREEDER BLANKET OF ITER
}

\author{
Heng Xie \\ Institute of Nuclear Energy technology, \\ Tsinghua University \\ xheng@mail.tsinghua.edu.cn
}

\author{
Zhiwei Zhou \\ Institute of Nuclear Energy technology, \\ Tsinghua University \\ zhouzhw@mail.tsinghua.edu.cn
}

\section{INTRODUCTION}

In China Helium-Cooled Solid Breeder Blanket (CH-HCSB), heat is carried out through Helium Cooling System (HCS) that consist of the cooling channels inside the First Wall (FW) and supporting structure of TBM, the main helium pipe connecting the TBM and Helium/Water heat exchanger, helium circulator, buffer volume and other auxiliary equipments. One of the accidents most possibly occurred in TBM is in vessel loss of coolant. In present study, the blowdown process of helium in HCS is theoretically modeled. The model is validated by comparison with numerical simulation results. The blowdown process is analyzed and the effect of friction is also studied.

\section{MODEL}

Since the structure of HCS is too complicated to be completely theoretically modeled, it is simplified as Fig.1: the analyzed volume is divided into three sub-volumes: Volume 1(HCS, not including cooling channels in TBM), Volume 2 (including cooling channels in TBM) and Volume 3

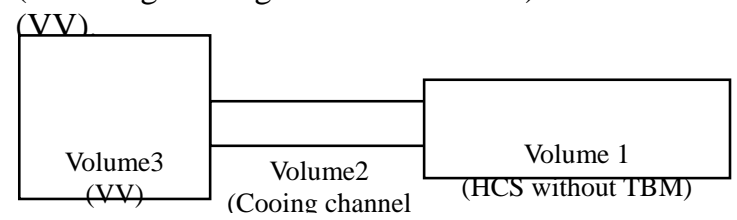

Fig1 Schematic of volume division

The volume of Volume 1 and Volume 3 are far large than that of Volume 2 and the friction in Volume 1 and Volume 3 are far smaller than that in Volume 2 due to thin channels in TBM. Therefore, it is assumed that all pressure loss occurs in Volume 2 and interfaces between Volumes. The properties in Volume 1 and Volume 3 are assumed to be uniform and lumped parameter method is employed in analysis of these two Volumes.

After deduction, the correlation of the pressure in Volume 1 can be obtained as follow,

$$
\begin{aligned}
& p_{1}=\frac{p_{0}}{T_{0}^{C_{B}+1}} T^{C_{B}+1}= \\
& \frac{p_{0}}{T_{0}^{C_{B}+1}}\left(T_{0}^{-0.5}+\frac{C_{A}}{2} t\right)^{-2\left(C_{B}+1\right)}
\end{aligned}
$$

The mass and pressure of helium in Volume 3 can also be obtained

$m_{3}=\frac{P_{0}}{R T_{0}^{C_{B}+1}} V_{1}\left[T_{0}^{C_{B}}-\left(T_{0}^{-0.5}+\frac{1}{2} C_{A} t\right)^{-2 C_{B}}\right]$

$$
\begin{aligned}
& P_{3}=\left[k+\frac{1}{2} M^{2} k(k-1)\right] \times \\
& {\left[T_{0}^{C_{B}+1}-\left(T_{0}^{-0.5}+\frac{1}{2} C_{A} t\right)^{-2 C_{B}-2}\right] \frac{P_{0} V_{1}}{T_{0}^{C_{B}+1} V_{3}}}
\end{aligned}
$$

\section{VALIDATION AND ANALYSIS}

To validate above analytical correlations, the blowdown process after double-ended rupture of nine cooling channels in First Wall of TBM is calculated and compared with the numerical simulation results of three-dimensional general commercial computational fluid dynamic software FLUENT. Good agreements are obtained.

The blow-down process in TBM is simulated and analyzed. The results show that the flow in HCS keeps a high value in 20 seconds after break and the shock in vacuum vessel caused by pressure rising is not significant. The results also show that the flow friction plays important role in the blow-down process.

Copyright (C) 2007 by JSME 


\section{THEORETICAL ANALYSIS OF BLOWDOWN PROCESS IN CHINA HELIUM-COOLED SOLID BREEDER BLANKET OF ITER}

\author{
Heng Xie \\ Institute of Nuclear Energy technology, \\ Tsinghua University \\ Beijing, 100084, P.R.China \\ Phone:+86-10-62783555, \\ Fax:+86-10-62771150 \\ e-mail:xheng@mail.tsinghua.edu.cn
}

\author{
Zhiwei Zhou \\ Institute of Nuclear Energy technology, \\ Tsinghua University \\ Beijing, 100084, P.R.China \\ Phone:+86-10-62784826 \\ Fax:+86-10-62771150 \\ e-mail: zhouzhw@mail.tsinghua.edu.cn
}

Keywords: ITER, TBM, Blow-down, Theoretical analysis

\begin{abstract}
Blowdown process of helium in HCS of CH-HCSB is theoretically analyzed. The influenced area is divided into three volumes and uniform assumption is adopted for $\mathrm{VV}$ and main loop of HCS. Analytical correlations of mass and pressure in VV and HCS are obtained. Validation of the analytical model is done by comparison with the numerical results of 3D CFD code. The effect of flow friction is also analyzed. It is found that the flow friction plays important role in the blowdown process and cannot be omitted. The blowdown process in TBM is simulated and analyzed. The results show that the flow in HCS keeps a high value in 20 seconds after break, which will benefit to the removal of decay heat. The results also show that the shock in vacuum vessel caused by pressure rising is not significant.
\end{abstract}

\section{INTRODUCTION}

One of the main missions of International Thermonuclear Experimental Reactor (ITER) is to test the function and reliability of various breeder blanket modules to explore their potential for the demonstration plant. The functions of these Test Breeder Modules (TBMs) include shielding the vacuum vessel and superconducting coils, testing tritium breeder and structure material and remove heat from plasma (Boccaccini et al., 2001). Various TBMs are developed by the participating parties. In China Helium-Cooled Solid Breeder Blanket (CH-HCSB) (Fig.1)(SWIP, 2005), heat is carried out through Helium Cooling System (HCS) that consist of the cooling channels inside the First
Wall (FW) and supporting structure of TBM, the main helium pipe connecting the TBM and Helium/Water heat exchanger, helium circulator, buffer volume and other auxiliary equipments (Fig.2). The safety requirement from ITER is to ensure that the TBMs do not adversely affect the safety of ITER (Boccaccini et al., 2001a, 2001b; SWIP, 2005). One of the accident most possibly occurred in TBM is in vessel loss of coolant: multiple break of TBM FW cooling channels cause the high pressure helium coolant in HCS blowdown into the vacuum vessel (VV), subsequently the HCS is depressurized and VV is pressurized quickly (Bartels et al. 1996; Han, 2001; Sheng and Sjöberg, 2003; Tang and Parker,2003). The shock from the depressurization and pressurization, the heat

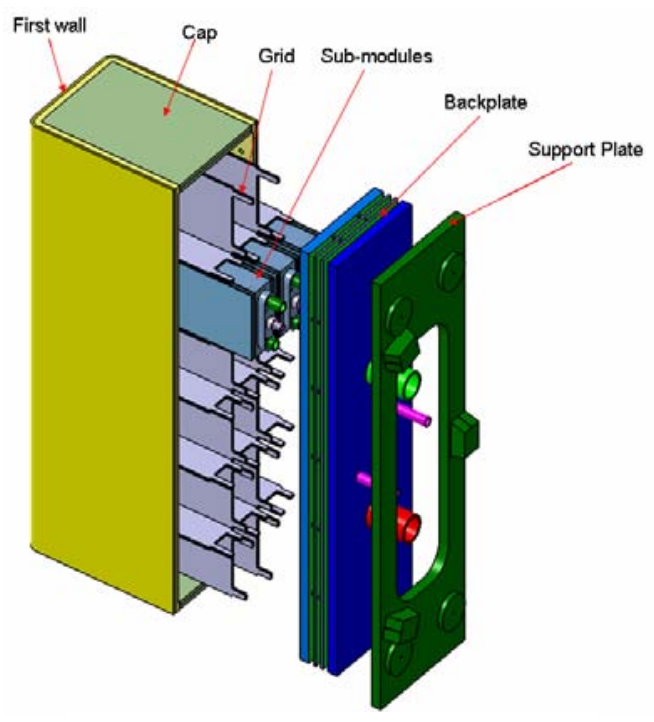

Fig.1 3D view of $C H-H C S B$ 

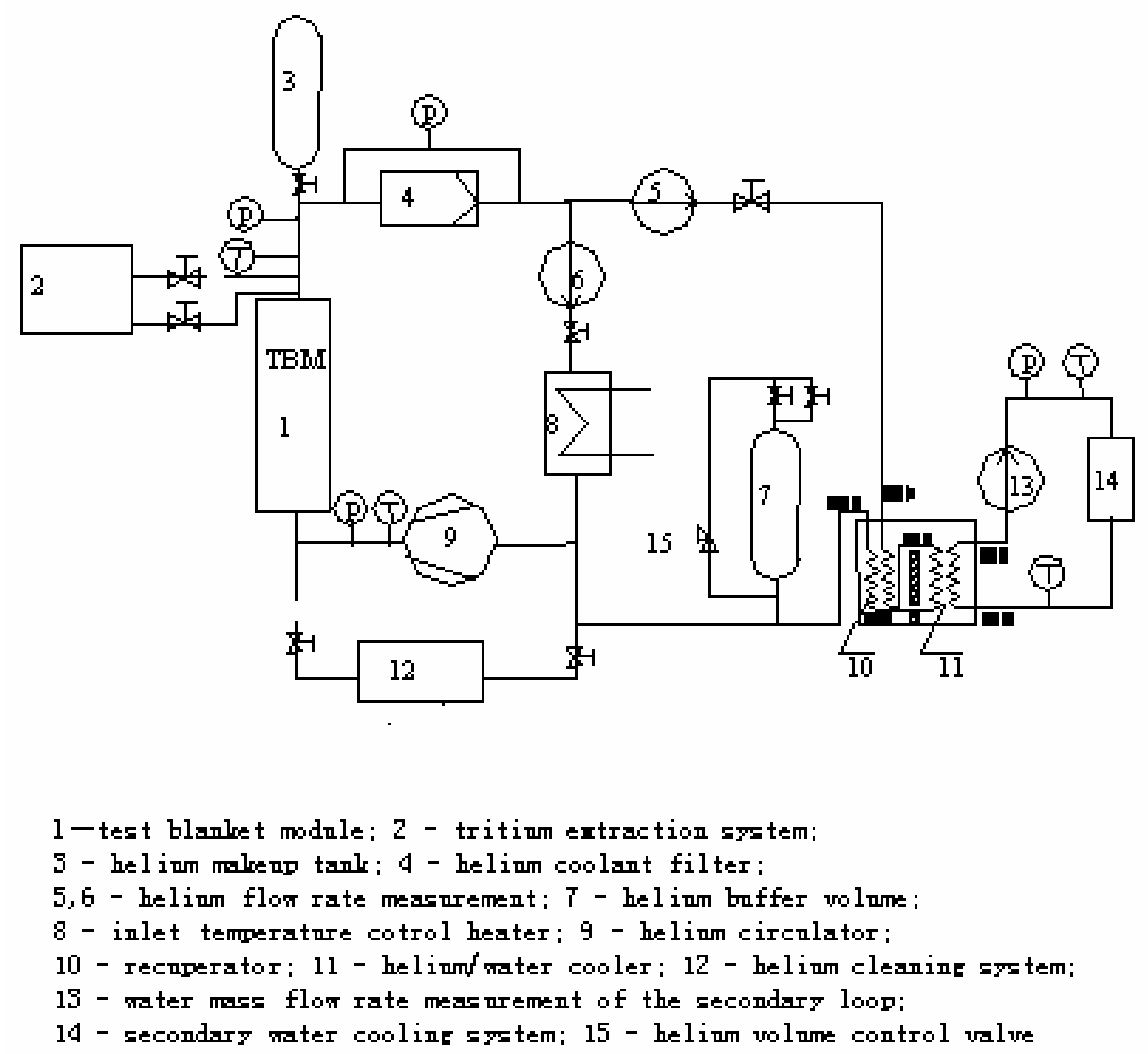

\section{Fig.2 Flow diagram of the CH-HCSB Helium Cooling System}

pulse resulted from plasma disruption and the decay heat in TBM will menace the safety of TBM and subsequently ITER. In present study, the blowdown process of helium in HCS is theoretically modeled. The model is validated by comparison with numerical simulation results. The blowdown process is analyzed and the effect of friction is also studied.

\section{MODEL}

Since the structure of HCS is too complicated to be completely theoretically modeled, it is simplified as Fig.3: the analyzed volume is divided into three sub-volumes: Volume 1(HCS, not including cooling channels in TBM), Volume 2 (including cooling channels in TBM) and Volume 3 (VV). The volume of Volume 1 and Volume 3 are far large than that of Volume 2 and the friction in Volume 1 and Volume 3 are far smaller than that in Volume 2 due to thin channels in TBM. Therefore, it is assumed that all pressure loss occurs in Volume 2 and interfaces between Volumes. The properties in Volume 1 and Volume 3 are

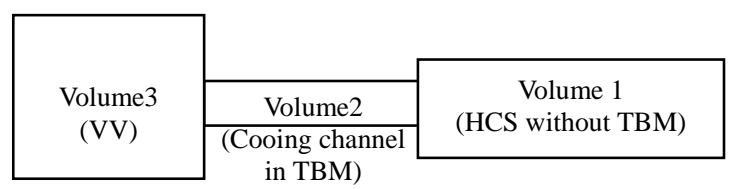

Fig.3 Schematic of volume division assumed to be uniform and lumped parameter method is employed in analysis of these two Volumes.

\subsection{Volume 2}

Since the flow of helium in cooling channels of TBM is very close to sound velocity, it is assumed to be adiabatic frictional Fano flow. Then the following conservation equations can be gotten for adiabatic flow in even channel.

Mass conservation equation:

$$
\rho u=C_{1}
$$

Energy conservation equation:

$$
h+u^{2} / 2=h_{0}
$$

Momentum conservation equation:

$$
d p+\rho u d u+\left(4 f d x / D+K_{L}\right) \frac{\rho u^{2}}{2}=0
$$

Ideal gas state equation: $\quad p=\rho R T$

Where, $\rho$ is density; $u$ is flow velocity; $h$ is enthalpy; $h_{0}$ is stagnant enthalpy; $p$ is pressure; $R$ is gas constant; $T$ is temperature; $f$ is friction coefficient ; $C_{1}$ is constant; $D$ is channel diameter; $x$ is channel length and $K_{L}$ is the local resistance coefficient.

The velocity of helium flow can be described by the product of Mach number and 
sound velocity,

$$
u=M \cdot C=M \sqrt{k R T}
$$

where $M$ is Mach number, $C$ is sound velocity and $\mathrm{k}$ is specific heat ratio.

Dividing the momentum Eq.3 by Eq.4, and replace velocity $u$ with Mach number $M$ (Eq.5), the following equation can be obtained:

$$
\frac{d p}{p}+k \frac{d M^{2}}{2}+\frac{k M^{2}}{2} \frac{d T}{T}+\frac{k M^{2}}{2}\left(4 f \frac{d x}{D}\right)=0
$$

From the ideal gas state equation, the following equation can be obtained:

$$
h=\frac{k R}{k-1} d T
$$

Substituting Eq.5 and Eq.7 into Eq.2 yields the following new energy equation,

$$
\frac{d T}{T}=-\frac{d\left(1+\frac{k-1}{2} M^{2}\right)}{1+\frac{k-1}{2} M^{2}}
$$

From Eq.1, Eq.4 and Eq.5, an equation of $P$, $T$, and $M$ can be obtained.

$$
\frac{d p}{p}=\frac{1}{2} \frac{d T}{T}-\frac{d M}{M}
$$

Since the volume of VV is far larger than that of HCS, even pressurized, its pressure will be far lower than HCS in a period after blowdown. Therefore, it can be assumed that the blowdown flow from HCS to VV keep critical flow in a short time and Mach number in the interface between Volume 2 and Volume 3 should be one.

Substituting Eq.8 and Eq.9 into Eq.6, and subsequently integrated over the cooling channel, an equation of Mach number in the interface between Volume 1 and Volume 2 can be obtained:

$$
\begin{aligned}
& \frac{4 f}{D} L+K_{L}=\frac{k+1}{2 k} \ln \left[M_{1,2}^{2}\left(\frac{\frac{k+1}{2}}{1+\frac{k-1}{2} M_{1,2}^{2}}\right)\right] \\
& +\frac{1}{k}\left(\frac{1}{M_{1,2}^{2}}-1\right)
\end{aligned}
$$

where $M_{1,2}$ is the Mach number of helium flow in the interface between Volume 1 and Volume 2,

$L$ is tube length.

\subsection{Volume 1}

Volume 1 is an open system and energy equation for open system is

$$
d E_{C V}=d\left(C_{V} \cdot m \cdot T\right)=\left(h+\frac{1}{2} u^{2}\right) d m
$$

where $E_{C V}$ is total inner energy of Volume, $C_{V}$ is constant volume specific heat.

From ideal gas state equation, equation of enthalpy and specific heat can be obtained:

$$
\begin{aligned}
& h=C_{P} T=\frac{k}{k-1} R T \\
& C_{V}=\frac{R}{k-1}
\end{aligned}
$$

The change of mass in Volume 1 is due to the outlet flow, so

$$
\begin{aligned}
& \frac{d m_{1}}{d t}=-\rho_{1} A_{1,2} u_{1,2} \\
& \text { And, } \\
& m_{1}=\rho_{1} \cdot V_{1}
\end{aligned}
$$

where $m_{1}$ is the helium mass in Volume $1, \rho_{1}$ is density of helium in Volume $1, A_{1,2}$ is interface surface between Volume 1 and Volume 2, $u_{1,2}$ is the helium flow velocity in the interface between Volume 1 and Volume 2 and $V_{1}$ is volume of Volume 1.

Substituting Eq.4, Eq.5 and Eq.12 15 into Eq.11, a simplified equation of Temperature in Volume1 can be obtained,

$$
\frac{d T_{1}}{d t}=-C_{A} T_{1}^{1.5}
$$

where

$$
C_{A}=\frac{A}{V} M_{1,2} \sqrt{k R}\left(1+\frac{1}{2} M^{2} k\right)(k-1)
$$

Integrating above equation with time, a correlation of temperature in volume $1, T_{1}$ can be obtained,

$$
T_{1}=\left(T_{0}^{-0.5}+\frac{1}{2} C_{A} t\right)^{-2}
$$

where $T_{0}$ is initial temperature of Volume 1.

Substituting Eq.4, Eq.15 and Eq.17 into Eq.11, the correlation of the helium mass in Volume 1 can be obtained,

$$
m_{1}=\frac{P_{0}}{R T_{0}^{C_{B}+1}} V_{1}\left(T_{0}^{-0.5}+\frac{1}{2} C_{A} t\right)^{-2 C_{B}}
$$

where $\mathrm{P}_{0}$ is initial pressure of Volume 1 and

$$
C_{B}=\frac{1}{\left(1+\frac{1}{2} M_{1,2}{ }^{2} k\right)(k-1)} \text {. }
$$

From ideal gas state equation $p V=m R T$, equation 17 and equation 18, the correlation of the pressure in Volume 1 can be obtained,

$$
\begin{aligned}
& p_{1}=\frac{p_{0}}{T_{0}^{C_{B}+1}} T^{C_{B}+1}= \\
& \frac{p_{0}}{T_{0}^{C_{B}+1}}\left(T_{0}^{-0.5}+\frac{C_{A}}{2} t\right)^{-2\left(C_{B}+1\right)}
\end{aligned}
$$




\subsection{Volume 3}

For Volume 3 (Vacuum Vessel), the change of mass and energy come from the helium flow from Volume 2. From energy equation of open system (Eq.11), we get the energy equation of Volume 3,

$$
\begin{aligned}
& d E_{C V 3}=d\left(C_{V} \cdot m_{3} \cdot T_{3}\right)= \\
& d m_{3}\left(h_{3}+\frac{1}{2} u_{2,3}{ }^{2}\right)
\end{aligned}
$$

Where $m_{3}$ is the mass of Volume $3, T_{3}$ is the temperature of Volume $3, h_{3}$ is the enthalpy of Volume 3 and $u_{2,3}$ is the helium flow velocity in the interface between Volume 2 and Volume 3.

The volume of Volume 2 is far less than that of Volume 1 and Volume 3. Therefore, it can be assumed that the change of helium in Volume 3 completely come from the change of helium in Volume 1,

$$
\begin{aligned}
& d m_{1}=-d m_{3} \\
& d m_{1}\left(h_{1}+\frac{1}{2} u_{1,2}^{2}\right)=-d m_{3}\left(h_{3}+\frac{1}{2} u_{2,3}^{2}\right)
\end{aligned}
$$

From Eq.18 and Eq.21, the mass of helium in Volume 3 can be obtained

$$
m_{3}=\frac{P_{0}}{R T_{0}^{C_{B}+1}} V_{1}\left[T_{0}^{C_{B}}-\left(T_{0}^{-0.5}+\frac{1}{2} C_{A} t\right)^{-2 C_{B}}\right]
$$

From Eq.4, Eq.17, Eq.20 and Eq.23, the correlation of the pressure in Volume 3 can be obtained,

$$
\begin{aligned}
& P_{3}=\left[k+\frac{1}{2} M^{2} k(k-1)\right] \times \\
& {\left[T_{0}^{C_{B}+1}-\left(T_{0}^{-0.5}+\frac{1}{2} C_{A} t\right)^{-2 C_{B}-2}\right] \frac{P_{0} V_{1}}{T_{0}^{C_{B}+1} V_{3}}}
\end{aligned}
$$

\section{VALIDATION AND RESULTS ANALYSIS}

To validate above analytical correlations, the blowdown process after double-ended rupture of nine cooling channels in First Wall of TBM is calculated and compared with the numerical simulation results. Numerical simulation are carried out by three-dimensional general commercial computational fluid dynamic software FLUENT. Nearly one million cells were employed. Adaptive time-step was used and time-step was very short due to the high velocity of helium flow. Therefore, the simulation of transient process is very time-consuming. The following parameters were employed in the calculation: the volume of Volume 1 is $2.39 \mathrm{~m}^{3}$, the volume of Volume 3 is $1350 \mathrm{~m}^{3}$, the length of cooling channel in TBM is
$0.5 \mathrm{~m}$, the diameter of cooling channel is $6 \mathrm{~mm}$, the initial temperature of Volume 1 is $375^{\circ} \mathrm{C}$ and initial pressure is $8 M P a$. Blasius correlation $f=0.079 / \mathrm{Re}^{0.25}$ is employed to calculate friction resistance.

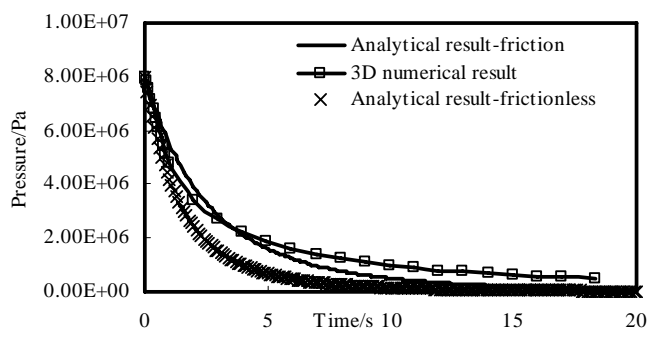

Fig.4 Time history of pressure in Volume 1of analytical and numerical results

The analytical and numerical simulation results of depressurization process of Volume 1 after blowdown are compared in Fig.4. To study the effect of friction, the frictionless analytical result is also shown in Fig.4. The results show that the depressurization process is very quick due to the high blowdown flowrate. The pressure of Volume 1 falls from $8 \mathrm{MPa}$ to less than 2MPa in 5 seconds. The friction analytical result is close to numerical result at the beginning, subsequently it will be less than that of numerical result. The reason can be explained as follow: at the beginning the pressure distribution in HCS is almost uniform and accord with the lumped analytical model; after several seconds, the pressure gradient in Volume 1 increase and the pressure in the outlet area will be distinctly less than the average value of Volume 1 . Therefore, the actual blowdown velocity that related to local pressure will be less than the analytical result and the actual depressurization process will be slower than the analytical result. Fig.4 also shows that the frictionless depressurization result is faster than friction result. The reason can be explained as follow: helium flow will reach critical in the interface between Volume 1 and Volume 2 if frictionless, whereas the friction helium flow will reach critical in the interface between Volume 2 and Volume 3 and the helium flow velocity in the interface between Volume 1 and Volume 2 will be less than sound velocity (calculated by Eq.10). The helium flow velocity is very high, this causes friction play important role in the blowdown and can not be omitted. The slower are the depressurization after blowdown, the more safe are the TBM. Therefore, the analytical result is more conservative than numerical result. Due to narrow discrepance between analytical and numerical result at the beginning and high time-consuming of numerical simulation, friction analytical model is employed in the following analysis.

Copyright $(2) 2007$ by JSME 


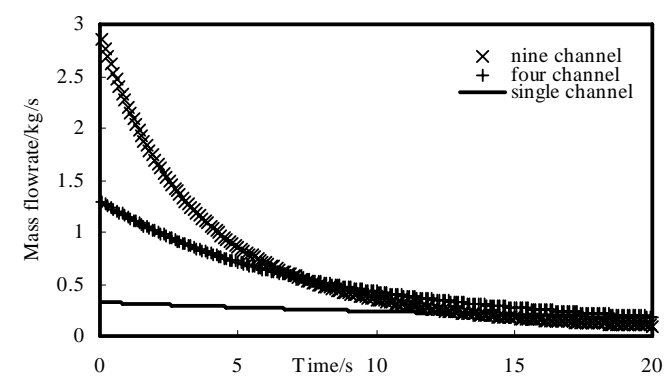

Fig.5 Time history of helium flow flowrate in break

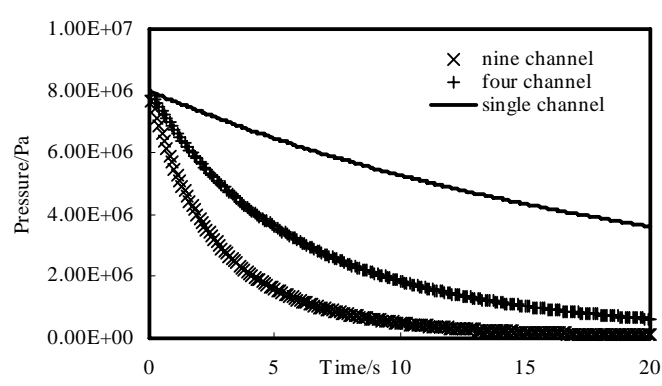

Fig.6 Time history of pressure in Volume 1

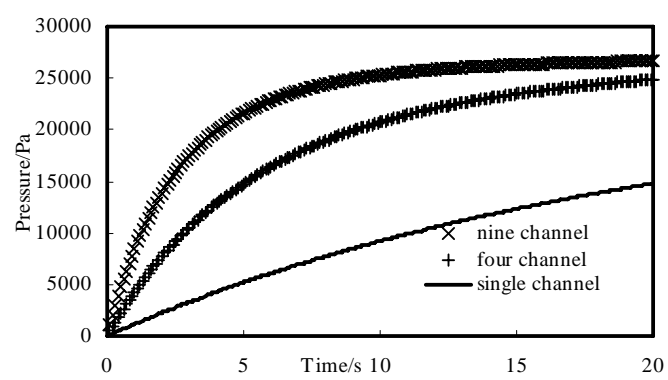

Fig.7 Time history of pressure in vacuum vessel

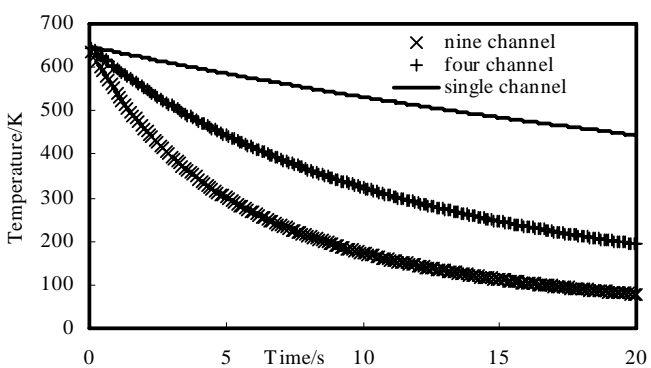

Fig.8 Time history of helium temperature in Volume 1

CHSB is divided into 9 parts and there are 9 $6 \mathrm{~mm}$-cooling channels in the first wall of each part. To study the effect of break size, blowdown process after double-ended rupture of one channel, 4 channels and 9 channels are analyzed. The calculated results of blowdown flowrate are shown in Fig.5. At the beginning, the flowrate are proportional to the break size due to same initial state. After $10 \mathrm{~s}$, the flowrate of 9 channels rupture become lower than other size due to its quick depressurization caused by great break flowrate at the beginning. The flowrate of all 3 sizes are higher than $0.1 \mathrm{~kg} / \mathrm{s}$ in $20 \mathrm{~s}$, which can insure the decay heat removal of CHSB. Fig.6 shows the depressurization process of Volume 1. The depressurization of 9 channel rupture is very fast due to big break size and pressure fall to atmosphere condition at 20s. The depressurization of single channel rupture is comparative slow and pressure fall to about $4 \mathrm{MPa}$ at 20s. The pressurization processes of vacuum vessel are shown in Fig.7. The VV pressure of 4 channel and 9 channel reach about $25 \mathrm{KPa}$ at 20s, which is still far less than the pressure in Volume 1. This validates the critical -flow hypothesis of analytical model. The maximum pressurization velocity occurs at the beginning and is only about $10 \mathrm{KPa} / \mathrm{s}$. Therefore, the shock to vacuum vessel structure caused by pressurization is slight and can be omitted. Fig.8 shows the time history of helium temperature in Volume 1. Since helium leaving Volume 1 in high velocity carry out a big quantity of energy, the inner energy of helium remaining in Volume 1 remarkably fall. The helium left expand to fill up the space. The expansion cause that the helium temperature reduce to a low level, especially in the 9-channel case. On the one hand, the reduce of helium temperature will benefit to the removal of decay heat; but on the other hand, too quick fall of temperature may destroy the structure material of TBM. The effect of temperature change to material should be studied later.

\section{CONCLUSION}

A theoretical model of blow-down process of helium in HCS of CH-HCSB is built and analytical correlations of mass and pressure in VV and HCS are obtained. Validation of the analytical model is done by comparison with the numerical results. The blow-down process in TBM is simulated and analyzed. The results show that the flow in HCS keeps a high value in 20 seconds after break and the shock in vacuum vessel caused by pressure rising is not significant. The results also show that the flow friction plays important role in the blow-down process.

The temperature in the TBM falls very quickly after blowdown and may destroy the structure material of TBM. The effect of temperature change to material should be studied in the future.

\section{REFERENCES}

Boccaccini, L. V., et al., 2001, Design Description Document for the European Helium Cooled Pebble Bed (HCPB) Test Blanket Modules. 
Boccaccini, L. V., et al., 2001, Safety assessment of ITER Test Blanket Modules.

Bartels, et al., 1996, Fusion Engineering and Design Vol. 31, 203.

Han, W. E., 2001, Fusion Engineering and Design Vol. 54, 413.

Sheng, CH. and Sjöberg, A., 2003, Fusion Engineering and Design Vol. 69, 577.
Southwestern Institute of Physics (SWIP), 2005, Design Description Document for the China Helium Cooled Solid-Breeder Test Blanket Module.

Tang V and Parker RR, 2003, Fusion Engineering and Design Vol. 65, 11. 